\title{
Killing Imaginary Numbers? From Today's Asymmetric Number System to a Symmetric System
}

\author{
Espen Gaarder Haug1, Pankaj Mani² \\ ${ }^{1}$ Norwegian University of Life Sciences, Ås, Norway \\ ${ }^{2}$ Independent Researcher, New Delhi, India \\ Email: espenhaug@mac.com
}

How to cite this paper: Haug, E.G. and Mani, P. (2021) Killing Imaginary Numbers? From Today's Asymmetric Number System to a Symmetric System. Advances in Pure Mathematics, 11, 741-754. https://doi.org/10.4236/apm.2021.118049

Received: July 8, 2021

Accepted: August 24, 2021

Published: August 27, 2021

Copyright () 2021 by author(s) and Scientific Research Publishing Inc. This work is licensed under the Creative Commons Attribution International License (CC BY 4.0).

http://creativecommons.org/licenses/by/4.0/ (c) (i) Open Access

\begin{abstract}
In this paper, we point out an interesting asymmetry in the rules of fundamental mathematics between positive and negative numbers. Further, we show an alternative numerical system identical to today's system, but where positive numbers dominate over negative numbers. This is like a mirror symmetry of the existing number system. The asymmetry in both systems leads to imaginary and complex numbers. We also suggest an alternative number system with perfectly symmetrical rules-that is, where there is no dominance of negative numbers over positive numbers or vice versa, and where imaginary and complex numbers are no longer needed. This number system seems to be superior to other numerical systems, as it brings simplicity and logic back to areas that complex rules have dominated for much of the history of mathematics. Finally, we also briefly discuss how the Riemann hypothesis may be linked to the asymmetry in the current number system. The foundation rules of a number system can, in general, not be proven incorrect or correct inside the number system itself. However, the ultimate goal of a number system is, in our view, to describe nature accurately. The optimal number system should therefore be developed with feedback from nature. If nature, at a very fundamental level, is ruled by symmetry, then a symmetric number system should make it easier to understand nature than an asymmetric number system would. We hypothesize that a symmetric number system may thus be better suited to describing nature. Further, such a number system should eliminate imaginary numbers in space-time and quantum mechanics, for example, two areas of physics that are clouded in mystery to this day.
\end{abstract}

\section{Keywords}

Asymmetry, Symmetry, Imaginary Numbers, Quantum Physics, Riemann 
Hypothesis

\section{Imaginary Numbers: Was Their "Discovery" a Sophisticated Innovation to Hide the Fundamental Inconsistency of Algebra?}

The history of imaginary numbers is an interesting one [1] [2]. Here, we will investigate how imaginary numbers are rooted in the asymmetry of our number system and that there could be other alternative number systems that give new light on imaginary numbers and, therefore, also potential on problems related to them. However, first, we will start with a quote from Freeman Dyson:

"No one [number] system is optimal. Different number systems are useful for different purposes. Freeman Dyson", Nov. 29, 2019”.

The early days of mathematics were only concerned with the arithmetic of positive numbers. Over time, ancient mathematicians conceived of negative numbers in their mental journey, and in confronting such numbers, the rules for mathematical operations were adjusted. As we begin exploring the properties of numbers and number systems, let us return to the foundation of mathematics again. What is positive and what is negative in the context of arithmetic? These are basic conventions of directions along a number line and are attached to some magnitudes like vectors. Positive and negative are relatively well-defined conventions with respect to each other, taking the origin on a basic $x-y$ graph as the reference point, i.e., numeric " 0 ".

As the study of mathematics progressed through the Middle Ages, more complex operations and concepts, some of which had emerged in earlier periods, were given greater attention. Square roots of negative numbers, for example, appeared in Ars Magna by Girolamo Cardano in 1545, who considered several forms of quadratic equations (e.g., $x^{2}+p x=q, \quad p x-x^{2}=q, x^{2}=p x+q$ ) simply to avoid using negative numbers. Cardano [3] said:

A second type of the false position makes use of roots of negative numbers. I will give an example: If someone says to you, divide 10 into two parts, one of which, multiplied into the other, shall produce 30 or 40 , it is evident that this case or question is impossible. Nevertheless, we shall solve it in this fashion. This, however, is closest to the quantity, which is truly imaginary since operations may not be performed with it as with a pure negative number, nor as in other numbers. This subtlety results from the arithmetic of which this final point is, as I have said, as subtle as it is useless.

Rafael Bombelli, in his book Algebra (1572), was more comfortable with negative numbers and addressed the rules of handling the signed quantities in the following manner:

${ }^{1}$ November 29, 2019, correspondence with Freeman Dyson concerning optimal number systems. Unfortunately, Dyson passed away just months later, February 28, 2020, but he was a great mathematical mind to the very end. 
- Plus times plus makes plus;

- Minus times minus makes plus;

- Plus times minus makes minus;

- Minus times plus makes minus.

Gauss, who gave a proof of the Fundamental Theorem of Algebra (1799), said in 1825 that "the true metaphysics of $\sqrt{-1}$ is illusive.", see for example [4]. Yet, by 1831 , he had overcome his doubts about applying complex numbers to number theory, which gave a tremendous boost to the acceptance of complex numbers in the mathematical community. Still, the acceptance was not universal. Augustus De Morgan, a famous mathematician, and logician wrote in 1831.

The imaginary expression $\sqrt{-a}$ and the negative expression $-b$ have this resemblance, that either of them occurring as the solution of a problem indicates some inconsistency or absurdity. As far as real meaning is concerned, both are equally imaginary since $0-a$ is as inconceivable as $\sqrt{-a}{ }^{2}$

It is clear that historically there has been a lack of universal consensus among mathematically-oriented scientists. In this paper, we examine this issue from both mathematical and philosophical perspectives. To begin, let us ask, "What does the square root of 4 mean?" And in contrast, "What does the square root of -1 mean at a fundamental level?"

The "square" term in square root was imported from geometry to arithmetic in ancient times. The square root itself means the length of each side of a square whose area might, for example, consist of four units. We know that the area of the square is equal to the length of one of the sides squared (and all sides are of equal length), so in this case, the length of each side is two units. Ideally, the sign assigned to the two here should correspond to the sign of the four, whether it is positive or negative (we are mapping the square on an $x-y$ graph here). If the area of square is positive with regard to the reference origin, then the length should be positive. Similarly, if the area of square is negative with regard to the reference origin, then the length should be negative. This is the logic from a real-world example, i.e., $\sqrt{+ \text { Number }}=+$ Number and $\sqrt{- \text { Number }}=-$ Number . In fact, the sign is not a geometrical figure, so we cannot take the square root of that in a true sense.

So, let us take a step back: will multiplying these things always lead to the square? For magnitudes, it will be true, but what about signs? As a step towards the answer, we can see that the current system takes $\sqrt{- \text { Number }}$ as being "Imaginary" and hence violating the logic drawn from geometry.

Another basic rule in mathematics is $\sqrt{a} \sqrt{b}=\sqrt{a \times b}$. This is defined for positive numbers, $a$ and $b$. However, if $a$ and $b$ are -1 , for example, then assuming this system is thoroughly consistent, $\sqrt{-1} \sqrt{-1}=\sqrt{-1 \times-1}=\sqrt{1}=1$. But with the definition of $\sqrt{-1}=i$, this leads to -1 , not 1 . This simple example shows how the earlier number system was incomplete. Then, to address this fundamental problem, mathematics was made unnecessarily complex in an effort to make it

\footnotetext{
${ }^{2}$ As cited in Kline [5], p. 593.
} 
complete.

Taking another simple example, the algebraic equation $x^{2}+4=3$, will lead to $x^{2}=3-4=-1$. This indicates that, given the concept of our defined rules of squaring, multiplication, addition, and subtraction, there is no value of $x$ possible here. So, we must either recheck and modify the incomplete assumptions made in the model/game, or we must conclude that no such $x$ exists in this system. Yet this truth has been hidden and forcibly made complete at the cost of introducing inconsistency and asymmetry into the system.

Confronted with this incompleteness in the rules of algebra defined for positive numbers, the long distant generation of mathematicians came up with $\sqrt{-1}$. They created a new convention to define this as an imaginary number $(i)$. It is a nice trick, but it also hides the fundamental and foundational incompleteness in the earlier defined rules of algebra. It was an attempt to create a new system where the relative nature of sign/direction (negative) could be transformed into an absolute number by defining $\sqrt{-1}$ as $(i)$ and building a new field of imaginary numbers.

We might ask, "Does the imaginary number try to model those non-observable aspects of nature mathematically?" But again, if this could be achieved at all, it would do so at the cost of compatibility between the incompleteness and asymmetry in the existing numerical system and the symmetry at a depth of reality in nature's system. One might note that many great mathematical minds have considered this tension between completeness and incompleteness; one of the highlights of the past 100 years is seen in the form of the Göedel Incompleteness Theorem (1931), which is considered to be one of the most powerful mathematical results of the 20th century.

It is also worth mentioning there is an asymmetry in exponential rules, where $2^{-1}=\frac{1}{2}$, while $2^{1}=2$ and $-2^{-1}=-\frac{1}{2}$ and $-2^{1}=-2$. In other words, asymmetry is found in several of the basic rules of mathematics.

Returning to our questions, the imaginary numbers are, in our view, created to make an asymmetrical system consistent. The output from such a number system, including imaginary numbers, can be difficult to interpret in terms of the physical world, especially when we are working at the deepest level of reality-the quantum level where we cannot even make direct observations, e.g., at the Planck scale [6] [7]. This means that such a numerical system can lead to strange and multiple interpretations, just as we see in mathematics and certain areas of physics today. This raises a fundamental question: Is this entire system of arithmetic consistent? Does it fall into line with nature's symmetries and principles? We think not, but what kind of system might take its place?

By introducing a symmetric number system, we will demonstrate that one gets totally rid of imaginary numbers, something that explains our title "Killing imaginary numbers." We hope this can motivate other researchers to see, for example, if this can lead to simpler interpretations in quantum mechanics and mathematical challenges where imaginary numbers today play an important role, 
and to some degree, put a mystical cloud around the interpretations. Imaginary numbers, we think, make it harder and less intuitive to interpret results, so getting rid of them can make us understand both mathematical and physical challenges better. This will, however, require further research along these lines.

\section{Perfect Symmetry}

The section above shows how negative numbers dominate over positive numbers in our current number system. We have also shown that there must exist an equivalent number system where positive numbers dominate over negative ones. These number systems are basically identical, or we could say they are mirror images of one other. Both number systems are asymmetric, and both need the concepts of imaginary and complex numbers to handle the square root function for all numbers.

Wouldn't it be nice to have a set of perfectly symmetrical mathematical rules that are identical for negative and positive numbers? This is also possible. We will suggest the following axioms:

1) A negative number multiplied with a negative number always gives a negative number.

2) A positive number multiplied with a positive number always gives a positive number.

3) A positive number multiplied with a negative number, or a negative with a positive number, always gives two solutions, namely a plus and minus solution of the absolute value of the result. However, one can select to only have the positive solution or only the negative solution. We could, for example, call the positive solution the principal multiplied solution.

4) $a^{b}$ is as in the standard number system, but $a^{-b}=-a^{b}= \pm a^{b}$ and $-a^{-b}=-\left(a^{b}\right)$.

The fact that we now have two solutions rather than one (under axiom 3) may seem strange at first, but this will help us eliminate multiple solutions regarding the square root function. The square root of a positive number will now always be positive, and the square root of a negative number will always be negative. Only the square root of a plus/minus number will have a plus/minus solution, and the plus/minus solution means that $\sqrt{ \pm 4}=-2 \times 2$, since \pm 4 can only be created by multiplying a positive number with a negative number.

Table 1 shows the three number systems mentioned here. The left-hand column is today's number system. The middle column is the mirror number system of our current system. The right-hand column is the newly suggested perfect symmetric number system. Again, we ask: "What is the rationale behind having negative numbers dominating over positive ones, or positive numbers dominating over negative ones"? Such asymmetry rules do not sound logical or appear to have any fundamental reasoning behind them. The asymmetric rules are the main cause of complex mathematics, such as imaginary numbers and complex number theory. Our symmetric number system seems more logical and may open 
Table 1. This table summarizes three different number systems. The first one is today's number system, where negative numbers dominate over positive. The next one is the mirror image of that system, where positive numbers dominate over negative. The third system is a number system with perfect symmetry, where negative and positive numbers have the same status. Only in the first two asymmetric number systems do we need imaginary and complex numbers.

\begin{tabular}{|c|c|c|}
\hline $\begin{array}{l}\text { Today's number } \\
\text { system } \\
\text { Asymmetric rules }\end{array}$ & $\begin{array}{l}\text { Mirror of today's } \\
\text { system } \\
\text { Asymmetric rules }\end{array}$ & $\begin{array}{l}\text { Perfect Symmetry } \\
\text { Yin-Yang system }\end{array}$ \\
\hline$+x+=+$ & $+x+=-$ & $+x+=+$ \\
\hline$-x-=+$ & $-x-=-$ & $-x-=-$ \\
\hline$+x-=-$ & $+x-=+$ & $+x-= \pm$ \\
\hline \multirow[t]{3}{*}{$-x+=-$} & $-x+=+$ & $-x+= \pm$ \\
\hline & & $-\times+=+$, principal squared plus \\
\hline & & $-x+=-$, principal squared negative \\
\hline$\sqrt{+}=+$ principal root & $\sqrt{+}=i$ & $\sqrt{+}=+$ \\
\hline$\sqrt{-}=i$ & $\sqrt{-}=-$ principal root & $\sqrt{-}=-$ \\
\hline$\sqrt{ \pm}=?$ No rule & $\sqrt{ \pm}=?$ No rule & $\sqrt{ \pm}=-x+$ \\
\hline \multicolumn{3}{|c|}{ Numerical examples } \\
\hline $2 \times 2=4$ & $2 \times 2=-4$ & $2 \times 2=4$ \\
\hline$-2 \times-2=4$ & $-2 \times-2=-4$ & $-2 \times-2=-4$ \\
\hline $2 \times-2=-4$ & $2 \times-2=4$ & $2 \times-2= \pm 4$ \\
\hline \multirow[t]{3}{*}{$-2 \times 2=-4$} & $-2 \times 2=4$ & $-2 \times 2= \pm 4$ \\
\hline & & $2 \times-2=4$ Principal solution \\
\hline & & $-2 \times 2=-4$ Principal negative solution \\
\hline$\sqrt{4}=2$ & $\sqrt{4}=2 i$ & $\sqrt{4}=2$ \\
\hline$\sqrt{-4}=2 i$ & $\sqrt{-4}=-2$ & $\sqrt{-4}=-2$ \\
\hline \multirow[t]{2}{*}{$\sqrt{ \pm 4}=?$} & $\sqrt{ \pm 4}=?$ & $\sqrt{ \pm 4}=\sqrt{-2} \sqrt{2}$ \\
\hline & & $\sqrt{ \pm 4}=\sqrt{2} \sqrt{-2}$ \\
\hline \multicolumn{3}{|c|}{ Addition and subtraction identical for all systems, as there is no sign dominance. } \\
\hline $2+2=4$ & $2+2=4$ & $2+2=4$ \\
\hline$-2+2=0$ & $-2+2=0$ & $-2+2=0$ \\
\hline $2-2=0$ & $2-2=0$ & $2-2=0$ \\
\hline$-2-2=-4$ & $-2-2=-4$ & $-2-2=-4$ \\
\hline
\end{tabular}

new possibilities in the field of mathematics, as well as other fields that rely heavily on the theory and practice of math. Obviously, as it is a new number system, there could be challenges that we have not anticipated. However, it is clear that small changes in the fundamental properties and rules of the prevailing number system can have a series of consequences for rules "higher" up in the constructions of math and physics, for example.

\section{Our $+x-= \pm$ Rule in Perspective}

Some will possibly object when we suggest that $+x-= \pm$. How can we have two 
solutions here? Before we answer that, let us look at the existing standard number system. Here, the rules for $+\times-=-$, so it always has one solution and seems simpler and more well-defined than our suggested rule. This is until we understand that we have the same issue in the standard number system; when working with the square root of a positive number, this gives two solutions. However, the way this rule is practiced in applied math would seem as if we could decide on the solution; we could simply ignore the negative solution, for example.

To illustrate the point, in finance, we can see that in many calculations in relation to uncertainty (for example, in many formulas in option pricing, see [8]), we have $\sqrt{T}$ where $T$ is the time to maturity on a financial contract. Assuming that there are four years to maturity on this contract, then $\sqrt{T}=\sqrt{4}=2$. That is, the negative solution is always ignored in this instance, as $\sqrt{T}$ is defined as the principal square; in other words, one excludes the negative solution of $\sqrt{-2 \times-2}=-2$, as time in these finance calculations cannot be negative. This is the case in a series of uncertainty calculations where $\sigma$ is the standardization and is then multiplied by the square root of time. Still imaginary numbers are also used actively in finance, see for example [9] [10]. However, this would also open the possibility for negative uncertainty, which makes no sense, except perhaps as a mathematical trick, see [11]. The same is true in physics. In Einstein's special relativity theory [12], for example, we often have the factor $\sqrt{1-\frac{v^{2}}{c^{2}}}$, where $v$ is the speed of the object $(v<c)$ and $c$ is the speed of light. In other words, the number inside the square root will always be $\geq 0$, and any negative solution to $\sqrt{1-\frac{v^{2}}{c^{2}}}$ will be ignored, again because one defines it as principal square, but in our view, the principal root is partly just an extra mathematical rule to fix a non-optimal foundation.

It appears that the standard number system gives us the freedom to choose whether we want to use both solutions or to select only one solution when we are dealing with $\sqrt{+}= \pm$ (that actually is written as $\pm \sqrt{+}= \pm$ ). In our suggested symmetric number system, the rules are clear cut for $\sqrt{+}=+$ and $\sqrt{-}=-$. The flexible rules that exist in the standard asymmetric number system for $\sqrt{+}= \pm$ that actually is written as $\pm \sqrt{+}= \pm$, have in our new symmetric number system been shifted to $+\times-= \pm$ instead. When we use only the positive solution, we could, for example, call it the principal solution, and when choosing only the negative solution, we can call it the principal negative solution so that one can describe it also in words. So, in our system, within applications, we can choose between the plus or minus solution, or we can use both, depending on what is applicable in the specific case. Further, we cannot see how this rule leads to any new issues, except that the conventional way, which we are all accustomed to, says that we will use the flexible rule for $\pm \sqrt{+}= \pm$ rather than for $+\times-= \pm$. The already flexible rules of choosing between a positive and 
negative solution are simply moved from one place in the standard number system (linked to square roots) to another place in our symmetric number system (linked to multiplications of negative and positive numbers).

However, by moving the flexible rule from $\pm \sqrt{+}= \pm$ to $+x-= \pm$, we discover one great advantage over the existing number system, namely that we have eliminated imaginary numbers, and also that the rules in our system are fully symmetrical.

\section{The Riemann Hypothesis and Its Possible Link to Asymmetric Number Systems}

One of the most interesting mathematical problems yet to be solved is the Riemann hypothesis. It is one of the seven "Millennium" Problems described by the Clay Mathematics Institute (CMI) and the only problem remaining from David Hilbert's original set of 23 problems, curated and presented in 1900, see [13] [14]. Solving the Riemann hypothesis has many important implications, potentially also for our understanding of areas in physics, see [15] [16] [17].

As described by the CMI, the Riemann hypothesis states that "some numbers have the special property that cannot be expressed as the product of two smaller numbers, e.g., 2, 3, 5, 7, etc. Such numbers are called prime numbers, and they play an important role, both in pure mathematics and its applications. The distribution of such prime numbers among all natural numbers does not follow any regular pattern. However, the German mathematician G.F.B. Riemann (1826-1866) observed that the frequency of prime numbers is very closely related to the behavior of an elaborate function, called the Riemann Zeta function. The Riemann hypothesis asserts that all interesting solutions of the equation $\zeta(s)=0$ lie on a certain vertical straight line. This has been checked for the first 10,000,000,000,000 solutions. A proof that it is true for every interesting solution would shed light on many of the mysteries surrounding the distribution of prime numbers."

Interestingly, there can be no (standard) Riemann hypothesis in our new symmetric number system, as there are no imaginary numbers and complex planes in this system. In other words, the Riemann hypothesis seems to be linked to and perhaps may even arise from the asymmetry in today's number system. In addition, we conjecture that the Riemann Zeta function, if developed under the mirror system (where the rules of dominant-negative number rules are switched, so positive number rules are dominant), what we could call the mirror Riemann Zeta function, should have all of its non trivial zeros only at $-1 / 2$ rather than at $1 / 2$ as in todays number system. In other words, we suggest that the Riemann hypothesis is partly rooted in the choice of the asymmetric number system. We have two asymmetric number systems that mirror each other, reflecting the Riemann hypothesis around zero ${ }^{4}$. Further, it appears that we have one symmetric number system with no equivalent Riemann hypothesis (but perhaps one can be

${ }^{3}$ See the general entry for the Riemann hypothesis on the clay mathematics institute website at: http://www.claymath.org/millennium-problems/riemann-hypothesis. 
developed also inside the symmetric number system). This leads us to think that the Riemann hypothesis is mostly about understanding the complex effects of a fundamental issue rooted in asymmetric rules between positive and negative numbers.

Even if the Riemann hypothesis should be solved inside the standard number system, our new number system could potentially give additional insight into the problem or at least the origin of the problem. This naturally needs to be investigated carefully before any conclusions are made, something hat we will leave for another time or other researchers to thoroughly investigate.

\section{How to Decide on an Optimal Number System}

A number system is similar to a set of rules for a game, like chess, where the game rules are about numbers themselves. Yet, the rules of a number system cannot, in general, be proven inside that number system. For example, one cannot prove or disprove that $-2 \times 2=-4$ is incorrect or correct; it is correct inside the system's rules. However, as we have seen, the asymmetric rules in the standard number system lead to the fact that one must introduce strange rules such as imaginary numbers. If the rules are defined, and the system is just used to solve purely mathematical problems inside the numerical system, nothing is wrong with this. It is like checking out all the rules in the system and playing around with them with many types of scenarios allowed inside the system. It is even possible to add new rules along the way when new challenges inside the system arise, such as finding the square root of minus one.

However, one of the ultimate goals of mathematics should be developing a language to describe better and understand nature. Here, even if the optimal rules cannot be proven right or wrong, they should emerge out of feedback from nature. For example, suppose one discovers that the rules of nature seem to be symmetrical rather than asymmetrical. In that case, one should reconsider the approach to mathematical rules that would make them symmetrical and therefore more harmonious with what one is describing. In other words, (and paraphrasing Einstein), one wants the numerical system to be as simple as possible so that it can describe nature, but not simpler than that. For perspective, that we must rely on imaginary numbers in quantum mechanics seems a bit strange. What would quantum mechanics look like if it was derived from a symmetric number system? To find the optimal number system, we need to evaluate it in relation to nature and see if that system seems better suited than another system with different rules.

In Table 2, we mention a few more possible number systems that are symmetrical. The system in column 1 is a very interesting alternative. Systems 3 and 4 could also be fully usable, but they seem to add complexity to thinking, e.g., why should one times one become minus one when we can have one times one as simply one? Still, any symmetric number system seems to get rid of imaginary ${ }^{4}$ And it is suggested that all non-trivial solutions are at $1 / 2$ in the standard asymmetric number system and at $-1 / 2$ in the mirror asymmetric number system. 
Table 2. This table summarizes three different symmetric number systems. However, these seems to add complexity in logic relative to the symmetric number system introduced in Table 1. Still any symmetric number system seems to get rid of imaginary numbers.

\begin{tabular}{lll}
\hline \multicolumn{1}{c}{$\begin{array}{c}\text { Symmetric system } 2 \\
\text { Yin-Yang system } 2\end{array}$} & Symmetric system 3 & \multicolumn{1}{c}{ Symmetric system $4^{\mathrm{a}}$} \\
\hline$+\times+=+$ & $+\times+=-$ & $+\times+=-$ \\
\hline$-\times-=-$ & $-\times-=+$ & $-\times-=+$ \\
\hline$+\times-=$ Not allowed, needed? & $+\times-= \pm$ & $+\times-=$ Not allowed, needed? \\
\hline$-\times+=$ Not allowed, needed? & $-\times+= \pm$ & $-\times+=$ Not allowed, needed? \\
\hline$\sqrt{+}=+$ & $\sqrt{+}=-$ & $\sqrt{+}=-$ \\
\hline$\sqrt{-}=-$ & $\sqrt{-}=+$ & $\sqrt{-}=+$ \\
\hline & Numerical examples & \\
\hline $2 \times 2=4$ & $2 \times 2=-4$ & $2 \times 2=-4$ \\
\hline$-2 \times-2=-4$ & $-2 \times-2=4$ & $-2 \times-2=4$ \\
\hline $2 \times-2=$ Not allowed & $2 \times-2= \pm 4$ & $2 \times-2=$ Not allowed \\
\hline$-2 \times 2=$ Not allowed & $-2 \times 2= \pm 4$ & $-2 \times 2=$ Not allowed \\
\hline$\sqrt{4}=2$ & $\sqrt{4}=-2$ & $\sqrt{4}=-2$ \\
\hline$\sqrt{-4}=-2$ & $\sqrt{-4}=2$ & $\sqrt{-4}=2$ \\
\hline$\sqrt{ \pm 4}=$ Not allowed & $\sqrt{ \pm 4}=\sqrt{2} \sqrt{-2}$ & $\sqrt{ \pm 4}=$ Not allowed \\
\hline Addition and subtraction identical for all systems, as there is no sign dominance. \\
\hline $2+2=4$ & $2+2=4$ & $2+2=4$ \\
\hline$-2+2=0$ & $-2+2=0$ & $-2+2=0$ \\
\hline$-2-2=-4$ & $2-2=0$ & $-2=-4$ \\
\hline & $-2-2=-4$ & \\
\hline
\end{tabular}

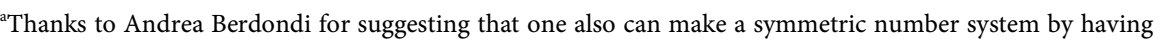
plus times plus is minus and minus times minus is plus. Still, this system appears to be more awkward and less logical than our Yin-Yang number systems.

numbers, so imaginary numbers seem to be a consequence of the historical choice of a asymmetric number system. Asymmetry in the rules in a number system forces one to invent imaginary numbers to keep the system consistent. Imaginary numbers are not wrong, as they are clearly needed and very useful, but they can be partly seen as a fix to get a asymmetric number system to work.

We think that our original Yin and Yang system (column 3, Table 1) might be the optimal number system to describe nature and simplify mathematics. However, more research is clearly needed to determine if this is the case. Also, one would likely need to develop mathematical software to handle our new symmetric number system to see more of the predictions it leads to.

\section{Imaginary Numbers in Physics}

In 1908, Minkowski [18] introduced imaginary numbers in his space-time geometry: 
Thus the essence of this postulate can be expressed mathematically very concisely in the mystical formula: $3 \times 10^{5} \mathrm{~km}=\sqrt{-1}$ seconds .

According to Unruh [19], whether or not Minkowski space-time is compatible with quantum theory is still an open question. There are also various interpretations of why imaginary numbers play an important role in Minkowski space [20] [21]. The imaginary number $\sqrt{-1}=i$ plays a central part in standard quantum mechanics [22] [23] [24]. We find it in the mechanics of Born and Jordan, then the Poisson bracket of Dirac and the Schrödinger equation [25], and later the Feynman's path integral, see [26] [27] [28]. It appears that it is indispensable in the formulation of quantum mechanics; see also [29]. Freeman Dyson, in a recent lecture, stated (see [30]).

"Surely, to the unpreoccupied mind, complex numbers are far from natural or simple, and they cannot be suggested by physical observations. Furthermore, the use of complex numbers is, in this case, not a calculational trick of applied mathematics but comes close to being a necessity in the formulation of the laws of quantum mechanics. But then came the surprise. Schrödinger put the square root of minus one into the equation, and suddenly it made sense. Suddenly it became a wave equation instead of a heat conduction equation. And that square root of minus one means that nature works with complex numbers and not with real numbers."

In a recent paper, Renou et al. [31] discuss the importance of complex numbers in relation to Bell's theorem. An interesting question is how the interpretation would be if we switched to our symmetric number system. Further, Bell's theorem relies on the Heisenberg uncertainty principle as always holding, even at the Planck scale, something that has been contested recently by Haug [32] [33] [34].

In our view, we have reasons to think that nature does not work with complex numbers, but researchers use a perhaps unnecessarily complex asymmetric number system that could be replaced with a symmetric number system that potentially better describes nature. We encourage future research to determine whether or not a new quantum mechanics rooted in our symmetric Yin and Yang number system could be developed. In this framework, clearly, no imaginary number can exist. If such a theory then still fits both classic experiments and modern quantum mechanics, then it would be preferable, as it would be more logical. After all, what does an imaginary number in an equation representing the real world truly represent? Truly, we would say, nothing but an overly complex model used to describe a simpler and perhaps more symmetrical reality. However, no conclusions should be drawn prematurely before this proposal is carefully investigated, and this is likely to take a considerable amount of time.

\section{Conclusions}

We have pointed out that our modern number system has "strange" asymmetric rules, where negative numbers dominate over positive numbers (when multip- 
lying positive numbers with negative). These asymmetric rules likely came into being because early mathematicians first developed rules for positive numbers and then tried to fit negative numbers into this system. The focus was to have a practical everyday number system. Later, it was necessary to develop a rule for the square root of numbers, which required some accommodation in the rules. The asymmetric rules seem to lead to the need for imaginary numbers. Further, we have shown that an identical or mirror number system exists, where the dominance rules are switched from negative numbers to positive ones. In this case, the imaginary numbers are linked to the square root of one rather than the square root of minus one.

We have also introduced a new perfectly symmetrical number system, where there is no dominance of negative over positive numbers or positive over negative numbers. In this perfectly symmetrical number system, there is no need for imaginary numbers or complex number theory. We also have indicated that the Riemann hypothesis is likely rooted in the asymmetry of the dominance rules in the existing number system. We hypothesize that a new and likely simpler quantum mechanics perhaps could be formulated based on our suggested symmetrical number systems. Thus, we have reasons to think nature does not consist of imaginary stuff but that the asymmetry in the current number systems needs to incorporate imaginary numbers to describe a deeper symmetry at a depth of reality; this naturally requires further investigation before any firm conclusions are made.

\section{Conflicts of Interest}

The authors declare no conflicts of interest.

\section{References}

[1] Nahin, P.J. (1998) An Imaginary Tale: The Story of $\sqrt{-1}$. Princeton University Press, Princeton.

[2] Nikouravan, M. (2019) A Short History of Imaginary Numbers. International Journal of Fundamental Physical Sciences, 9, 1-5.

https://journals.indexcopernicus.com/api/file/viewByFileId/659813.pdf

[3] Cardano, G. (1545) Ars Magna or The Rules of Algebra. Dover Publications, New York.

[4] Dodge, C. (2004) Euclidean Geometry and Transformations. Dover Publications, New York.

[5] Kline, M. (1972) Mathematical Thought from Ancient to Modern Times. Volume 2, Oxford University Press, Oxford.

[6] Planck, M. (1899) Natuerliche Masseinheiten. Der Königlich Preussischen Akademie Der Wissenschaften, Berlin.

[7] Planck, M. (1906) Vorlesungen über die Theorie der Wärmestrahlung. Dover Publications, New York, 163.

[8] Haug, E.G. (2007) The Complete Guide To Option Pricing Formulas. 2nd Edition, McGraw-Hill, New York.

[9] Svetunkov, S. (2012) Complex-Valued Modeling in Economics and Finance. Sprin- 
ger, Berlin. https://doi.org/10.1007/978-1-4614-5876-0

[10] Dallago, R. and Facchinetti, B.R., Dallago, G. and Facchinetti. S. (2021) Black's Model in a Negative Interest Rate Environment, with Application to OTC Derivatives. Computational Management Science, 2021.

[11] Haug, E.G. (2002) A Look in the Antimatter Mirror. Wilmott Magazine, Wiley Publishing, 38-42.

[12] Einstein, A. (1923) On the Electrodynamics of Moving Bodies. Annalen der Physik, 17, 891-921.

[13] Riemann, B. (1859) Ueber die Anzahl der Primzahlen unter einer gegebenen Grösse. Monatsberichte der Berliner Akademie. Gesammelte Werke, Teubner.

[14] Hilbert, D. (1902) Mathematical Problems. Bulletin of the American Mathematical Society, 80, 437-479.

[15] Leclair, A. (2013) An Electrostatic Depiction of the Validity of the Riemann Hypothesis and a Formula for the Nth Zero at Large N. International Journal of Theoretical Physics, 28, Article ID: 1350151. https://doi.org/10.1142/S0217751X13501510

[16] Wolf, M. (2020) Will a Physicist Prove the Riemann Hypothesis? Reports on Progress in Physics, 83, Article ID: 036001. https://doi.org/10.1088/1361-6633/ab3de7

[17] Barbarani, V. (2020) A Quantum Model of the Distribution of Prime Numbers and the RIEMANN hypothesis. International Journal of Theoretical Physics, 59, 2425-2470. https://doi.org/10.1007/s10773-020-04512-2

[18] Minkowski, H. (1908) Space and Time. In: Davis, F.A., Ed., The Principle of Relativity, Dover Publications, New York.

[19] Unruh, W.G. (2009) Chapter: Minkowski Space-Time and Quantum Mechanics. In: Petkov, V., Ed., Minkowski Spacetime: A Hundred Years Later, Springer, Berlin. https://www.springer.com/gp/book/9789048134748 https://doi.org/10.1007/978-90-481-3475-5_5

[20] Adamo, T.M. and Newman, E.T. (2010) The Real Meaning of Complex Minkowski-Space World-Lines. Classical and Quantum Gravity, 27, Article ID: 152011. https://doi.org/10.1088/0264-9381/27/7/075009

[21] Smith, F.T. (2012) A New View of Minkowski Space. Journal of Physics. Conference Series, 338, Article ID: 152011. https://doi.org/10.1088/1742-6596/388/15/152011

[22] Lev, F.M. (2006) Why Is Quantum Physics Based on Complex Numbers? Finite Fields and Their Applications, 12, 336-356. https://doi.org/10.1016/j.ffa.2005.07.006

[23] Hickey, A. and Gour, G. (2018) Quantifying the Imaginarity of Quantum Mechanics. Journal of Physics A: Mathematical and Theoretical, 51, Article ID: 414009. https://doi.org/10.1088/1751-8121/aabe9c

[24] Karam, R. (2020) Why Are Complex Numbers Needed in Quantum Mechanics? Some Answers for the Introductory Level. American Journal of Physics, 88, 39. https://doi.org/10.1119/10.0000258

[25] Schrödinger, E. (1926) An Undulatory Theory of the Mechanics of Atoms and Molecules. Physical Review, 280, 1049. https://doi.org/10.1103/PhysRev.28.1049

[26] Feynman, R. (1948) Space-Time Approach to Non-Quantum Mechanics. Review of Modern Physics, 20, 367. https://doi.org/10.1103/RevModPhys.20.367

[27] Feynman, R. and Hibbs, A. (1965) Quantum Mechanics and Path Integrals. McGraw Hill, New York.

[28] Niestegge, G. (2014) Composite Systems and the Role of the Complex Numbers in Quantum Mechanics. Journal of Mathematical Physics, 45, 4714. 
https://doi.org/10.1063/1.1811371

[29] Gull, S., Lasenby, A. and Doran, C. (1993) Imaginary Numbers Are Not Real-The Geometric Algebra of Spacetime. Foundations of Physics, 23, 1175-1201. https://doi.org/10.1007/BF01883676

[30] Kwong, C.P. (2009) The Mystery of Square Root of Minus One in Quantum Mechanics, and Its Demystification. ArXiV. https://arxiv.org/pdf/0912.3996.pdf

[31] Renou, M.O., et al. (2021) Quantum Physics Needs Complex Numbers. ArXiV. https://arxiv.org/pdf/2101.10873.pdf

[32] Haug, E.G. (2020) Collision Space-Time: Unified Quantum Gravity. Physics Essays, 330, 46.

[33] Haug, E.G. (2020) Rethinking the Foundation of Physics and Its Relation to Quantum Gravity and Quantum Probabilities: Unification of Gravity and Quantum Mechanics. https://doi.org/10.20944/preprints202012.0483.v1

[34] Haug, E.G. (2021) Quantum Gravity Hidden in Newton Gravity and How to Unify It with Quantum Mechanics. In: Krasnoholovets, V., Ed., The Origin of Gravity from the First Principles, NOVA Publishing, New York. 